CERN - PS DIVISION

CERN/PS 2001-049 (PP)

CERN NUFACT Note 083

\title{
STATUS OF STUDIES FOR A MUON COOLING EXPERIMENT
}

\author{
K. Hanke
}

\begin{abstract}
A possible layout for a cooling experiment is being studied to demonstrate the feasibility of the CERN reference scenario for a muon cooling channel of a future neutrino factory. Tracking of muons in 3D magnetic and electric fields was performed with the aim of validating the set-up, i.e. the possibility of extrapolating to the final reference channel. Simulation results are shown together with the cavity/solenoid engineering design. The direction of the work in progress is indicated and discussed.
\end{abstract}

Presented at NuFACT'01 Workshop, International Congress Centre, Tsukuba, Ibaraki, Japan

24-30 May 2001

Geneva, Switzerland

$15^{\text {th }}$ July 2001 


\section{INTRODUCTION}

The high neutrino flux needed for the physics at possible future neutrino factories requires about $10^{21}$ muons per year stored in the decay ring. In the CERN scheme, the muon recirculators and the decay ring have a transverse normalised acceptance of $1.5 \pi \mathrm{cm}$ rad. In order to collect the required number of muons inside this acceptance, transverse cooling of about a factor 20 is required. The cooling technique proposed is ionisation cooling, where muons pass through matter, loosing momentum in transverse and longitudinal direction. The longitudinal component is then replaced by rf cavities, thus leading to decreased angles. Multiple scattering in the absorber works agains the cooling process and determines a lower threshold (equilibrium emittance) that can be achieved.

\section{LAYOUT AND SIMULATION OF THE CHANNEL}

The CERN layout for a neutrino factory includes a cooling channel based on 44 and $88 \mathrm{MHz}$ cavities with integrated superconducting solenoids [1]. In order to prove the feasibility of such a cooling channel, a cooling experiment is proposed which is a small subset of the $88 \mathrm{MHz}$ part of the final channel. The system studied consists of a total of 16 cavities at $88 \mathrm{MHz}$ providing an average gradient of $4 \mathrm{MV} / \mathrm{m}$. For an input kinetic energy of $200 \mathrm{MeV}$, the energy lost by a length of $180 \mathrm{~cm}$ of liquid hydrogen absorber can be replaced by the cavities. The layout of the channel is such that there is a first absorber of $45 \mathrm{~cm}$ length at the entry of the cooling section, followed by two strings of 8 cavities with integrated solenoids, separated by a $90 \mathrm{~cm}$ central absorber. A third absorber of $45 \mathrm{~cm}$ length is placed at the exit of the cooling section. More recently, a scheme was studied which uses only half the number of cavities. The setup consists then of a $45 \mathrm{~cm}$ entry absorber, 8 cavity/solenoid structures and a $45 \mathrm{~cm}$ exit absorber. For both cases, the solenoidal lattice is continued upstream and downstream of the cooling cell where the input and output diagnostics are installed.

A beam dynamics study of the proposed cooling channel has been performed, based on the engineering design of cavities and solenoids. Cavities at $88 \mathrm{MHz}$ providing an average gradient of $4 \mathrm{MV} / \mathrm{m}$ have been designed [2] using SUPERFISH. The solenoids are integrated and have been designed [3] using ROXIE8.1. The solenoid design takes into account engineering constraints, such as space required for the cryogenic system and forces between solenoids. A maximum field strength of about $6 \mathrm{~T}$ on axis appears possible. The $3 \mathrm{D}$ electric and magnetic field maps have been merged and included in the tracking code PATH. The input beam parameters have been chosen close to those in the CERN reference cooling channel: $\beta=1 \mathrm{~m}, \alpha=0, \mathrm{E}_{\mathrm{kin}}=200 \mathrm{MeV}, \Delta \mathrm{E}= \pm 15 \mathrm{MeV}$. The solenoids were set to $6 \mathrm{~T}$ and the cavity synchronous phase to $0^{\circ}$. For this example, the transmission is $100 \%$ and the normalised r.m.s. emittance at the exit of the cooling channel is reduced by about $10 \%$ in each plane for the set-up with 16 cavities, and by about $5 \%$ per plane for the set-up with 8 cavities. Furthermore, the input emittance was varied in order to determine the response of the system. A scan of output versus input emittance is shown in Fig.1.

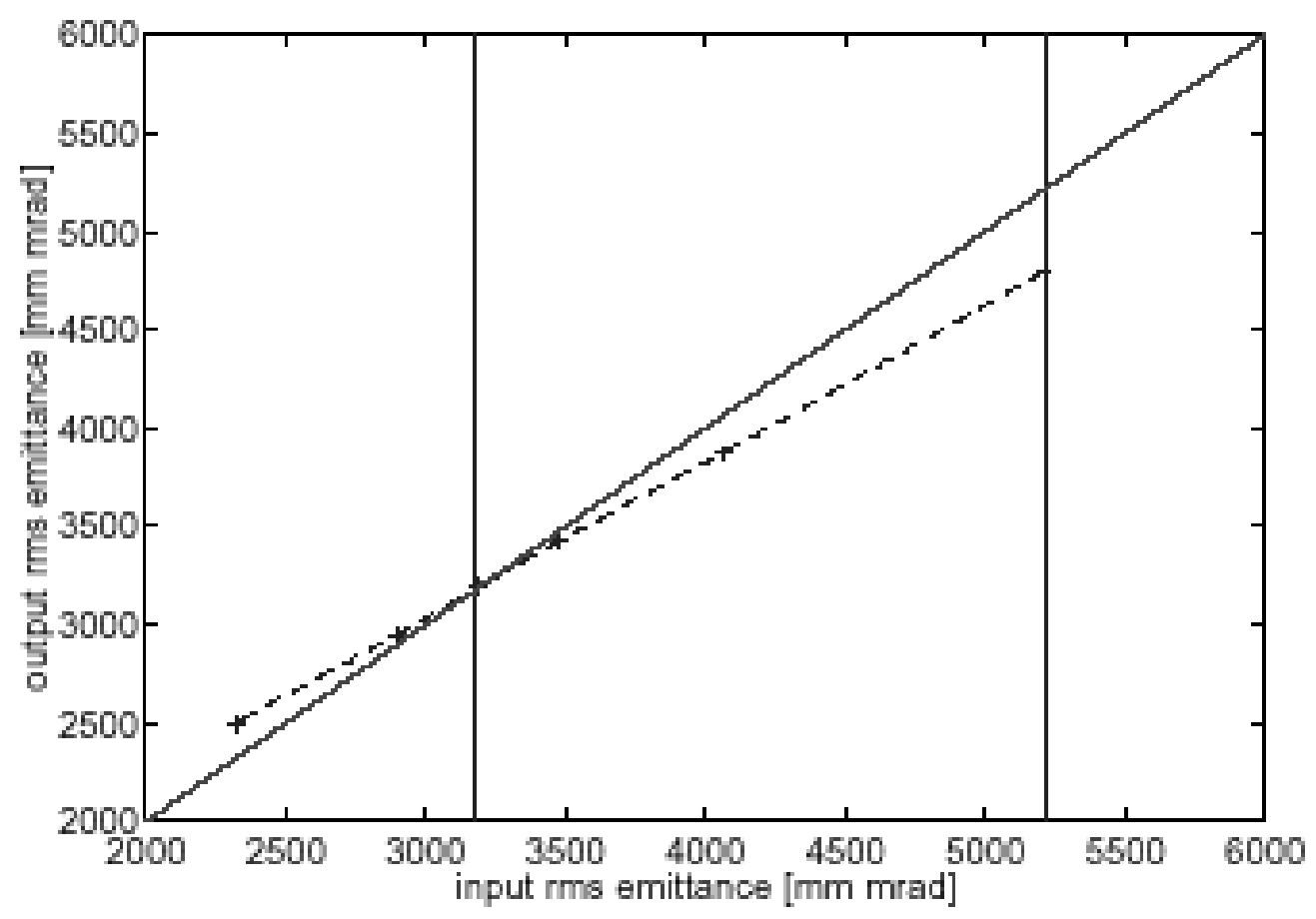

Fig 1. Scan of output versus input emittance for the set-up with 16 cavities. Dashed line: simulation results; solid line: $\varepsilon_{\text {in }}=\varepsilon_{\text {out }}$. The two vertical lines indicate the limits of the working regime: the lower limit is given by the equilibrium emittance, the upper limit by the acceptance of the channel. 


\section{EMITTANCE MEASUREMENT}

As traditional emittance measurements are limited to about $10 \%$ precision, it was proposed to measure positions and momenta of single particles inside a solenoid using scintillating fibre detectors [4]. While the positions are obtained directly from the detectors, the corresponding momenta have to be reconstructed from the trajectory of the particles in the solenoidal field. This method relies on a precise knowledge of the field map. Collecting sufficient statistics enables the whole beam to be reconstructed in phase space and to compute the beam emittance.

\section{CONCLUSION AND ONGOING WORK}

It has been shown as an example that from a small subset of the $88 \mathrm{MHz}$ CERN cooling channel and with engineered cavities and solenoids, one can expect an observable emittance reduction. For both the set-ups with 8 and 16 cavities, the parameter space is being explored and the settings of the channel optimised. Even a case with only 4 cavities will be investigated in order to identify the smallest possible experiment.Various optics, both with and without field flip, will be evaluated. A different way to analyse the results by counting particles inside a given 6D volume is being pursued.

\section{REFERENCES}

[1]. Lombardi, “A 40-80 MHz System for Phase Rotation and Cooling”, CERN Neutrino Factory Note 37 (2000).

[2] F. Gerigk, private communication.

[3] S. Russenschuck, M. Aleksa, private communication.

[4] P. Janot, private communication.

Preprint submitted to Elsevier Science 17 July 2001 